LPT-ORSAY 99/87

hep-th/9910219

\title{
Brane cosmological evolution in a bulk with cosmological constant
}

\author{
Pierre Binétruy ${ }^{1}$, Cédric Deffayet ${ }^{1}$, Ulrich Ellwanger ${ }^{1}$, David Langlois ${ }^{2}$ \\ ${ }^{1}$ LPT $\left.\right|^{\mid}$, Université Paris-XI, Bâtiment 210, F-91405 Orsay Cedex, France; \\ ${ }^{2}$ Département d'Astrophysique Relativiste et de Cosmologie, C.N.R.S., \\ Observatoire de Paris, 92195, Meudon, France.
}

\begin{abstract}
.
We consider the cosmology of a "3-brane universe" in a five dimensional (bulk) space-time with a cosmological constant. We show that Einstein's equations admit a first integral, analogous to the first Friedmann equation, which governs the evolution of the metric in the brane, whatever the time evolution of the metric along the fifth dimension. We thus obtain the cosmological evolution in the brane for any equation of state describing the matter in the brane, without needing the dependence of the metric on the fifth dimension. In the particular case $p=w \rho,(w=$ constant $)$, we give explicit expressions for the time evolution of the brane scale factor, which show that standard cosmological evolution can be obtained (after an early non conventional phase) in a scenario à la Randall and Sundrum, where a brane tension compensates the bulk cosmological constant. We also show that a tiny deviation from exact compensation leads to an effective cosmological constant at late time. Moreover, when the metric along the fifth dimension is static, we are able to extend the solution found on the brane to the whole spacetime.
\end{abstract}

\footnotetext{
${ }^{1}$ Unité mixte de recherche UMR n ${ }^{o} 8627$.
} 


\section{Introduction}

There has been recently a lot of activity on the possibility that we live in a threedimensional world embedded in a higher dimensional space. Modifying the old Kaluza-Klein picture [1], where the extra-dimensions must be sufficiently compact, these recent developpements are based on the idea that ordinary matter fields could be confined to a three-dimensional world, corresponding to our apparent Universe, while gravity could live in a higher dimensional space [2]. The usual constraints on Kaluza-Klein models could therefore be relaxed [3], and "large" extra-dimensions would be conceivable, thus leading to a fundamental Planck mass much lower than its apparent three-dimensional value, even as low as the $T e V$ scale. In the same spirit, Gogberashvili [4] and Randall and Sundrum [5], [6], reviving an older idea [7], have recently pointed out that the extra dimension (single in their case) need not even be compact.

A first important question concerning these kinds of models is to check whether they give back standard four-dimensional gravity in the brane assumed to represent our world. When the gravitational field generated by the brane itself is taken into account, it is not so easy to check that usual gravity is recovered. Randall and Sundrum showed that this is the case because of the existence of massless gravitons trapped in the brane, although small corrections are expected from massive modes due to the presence of a fifth dimension. Several groups have also begun to work on possible experimental signatures of these kinds of models [8].

In addition to the study of the weak field limit, one would expect that cosmology as well could provide constraints on these types of models. An interesting aspect of cosmology in such models is that, thanks to the symmetries, it is possible to deal with the non-linearities of the Einstein's equations and to detect effects that may not appear in a linearized treatment. The first crucial question is the viability of such models with respect to the cosmological evolution of our Universe. In a previous paper [9], three of us have shown that, generically, the equations governing the cosmological evolution of the brane will be different from the analogous Friedmann equations of standard cosmology (the same holds for a brane with finite thickness [10]). Essentially, the difference lies in the fact that the energy density of the brane appears quadratically in the new Friedmann equations in contrast with the linear behaviour of the usual equations. In the simplest model where the bulk is supposed to be empty (and stabilized), we found explicit solutions which showed that the corresponding cosmological scenario would be incompatible with nucleosynthesis constraints.

Following our work, it was noticed by two groups ([11], [12]) that a possible way to reconcile brane cosmology with the required standard cosmological scenario (at least since nucleosynthesis) would be to use Randall and Sundrum's approach [6], i.e. to put a (negative) cosmological constant in the bulk and to introduce in the brane, in addition to ordinary matter, a constant tension that would exactly 
compensate the bulk cosmological constant so that the, quadratic, leading order due to the brane contribution would be cancelled, leaving the next, linear, order.

These works however only provided an approximate perturbative analysis of the cosmological evolution. Now, exact solutions of the global Einstein's equations are extremely useful, and until now, such solutions with a bulk cosmological constant exist only when the spacetime is static ([5]), or in the extensions given by Kaloper [13 (see also [14] and [15]), where the brane tension does not exactly compensate the bulk cosmological constant, so that dynamical evolution is possible, but in the simplest form of exponential expansion. Some other exact solutions are also known in the context of general supergravity [16] or HoravaWitten supergravity [17]. General conditions for static universes can be found in [18].

The purpose of the present work is to solve the five-dimensional Einstein's equations for any type of matter in the brane with a cosmological constant in the bulk. We first show in section 2 that Einstein's equations admit a first integral, which in particular provides directly the cosmological evolution of the brane. In the following section, we show that an additional assumption, namely that the metric along the fifth dimension does not evolve in time, enables us to solve for the whole space-time metric, i.e. to find explicitly the dependence of the metric on the transverse coordinate as well as time. Finally, in the last section, we solve analytically, for different cases, the new Friedmann equation obtained in the present work and discuss the consequences.

\section{Solving Einstein's equations}

Let us present here the general framework. We shall consider five-dimensional spacetime metrics of the form

$$
d s^{2}=\tilde{g}_{A B} d x^{A} d x^{B}=g_{\mu \nu} d x^{\mu} d x^{\nu}+b^{2} d y^{2},
$$

where $y$ is the coordinate of the fifth dimension. Throughout this article, we will focus our attention on the hypersurface defined by $y=0$, which we identify with the world volume of the brane that forms our universe. Since we are interested in cosmological solutions, we take a metric of the form

$$
d s^{2}=-n^{2}(\tau, y) d \tau^{2}+a^{2}(\tau, y) \gamma_{i j} d x^{i} d x^{j}+b^{2}(\tau, y) d y^{2},
$$

where $\gamma_{i j}$ is a maximally symmetric 3 -dimensional metric $(k=-1,0,1$ will parametrize the spatial curvature).

The five-dimensional Einstein equations take the usual form

$$
\tilde{G}_{A B} \equiv \tilde{R}_{A B}-\frac{1}{2} \tilde{R} \tilde{g}_{A B}=\kappa^{2} \tilde{T}_{A B}
$$


where $\tilde{R}_{A B}$ is the five-dimensional Ricci tensor, $\tilde{R}=\tilde{g}^{A B} \tilde{R}_{A B}$ the scalar curvature and the constant $\kappa$ is related to the five-dimensional Newton's constant $G_{(5)}$ and the five-dimensional reduced Planck mass $M_{(5)}$, by the relations

$$
\kappa^{2}=8 \pi G_{(5)}=M_{(5)}^{-3} .
$$

Inserting the ansatz (2) for the metric, the non-vanishing components of the Einstein tensor $\tilde{G}_{A B}$ are found to be

$$
\begin{aligned}
\tilde{G}_{00}= & 3\left\{\frac{\dot{a}}{a}\left(\frac{\dot{a}}{a}+\frac{\dot{b}}{b}\right)-\frac{n^{2}}{b^{2}}\left(\frac{a^{\prime \prime}}{a}+\frac{a^{\prime}}{a}\left(\frac{a^{\prime}}{a}-\frac{b^{\prime}}{b}\right)\right)+k \frac{n^{2}}{a^{2}}\right\}, \\
\tilde{G}_{i j}= & \frac{a^{2}}{b^{2}} \gamma_{i j}\left\{\frac{a^{\prime}}{a}\left(\frac{a^{\prime}}{a}+2 \frac{n^{\prime}}{n}\right)-\frac{b^{\prime}}{b}\left(\frac{n^{\prime}}{n}+2 \frac{a^{\prime}}{a}\right)+2 \frac{a^{\prime \prime}}{a}+\frac{n^{\prime \prime}}{n}\right\} \\
& +\frac{a^{2}}{n^{2}} \gamma_{i j}\left\{\frac{\dot{a}}{a}\left(-\frac{\dot{a}}{a}+2 \frac{\dot{n}}{n}\right)-2 \frac{\ddot{a}}{a}+\frac{\dot{b}}{b}\left(-2 \frac{\dot{a}}{a}+\frac{\dot{n}}{n}\right)-\frac{\ddot{b}}{b}\right\}-k \gamma_{i j}, \\
\tilde{G}_{05}= & 3\left(\frac{n^{\prime}}{n} \frac{\dot{a}}{a}+\frac{a^{\prime}}{a} \frac{\dot{b}}{b}-\frac{\dot{a}^{\prime}}{a}\right), \\
\tilde{G}_{55}= & 3\left\{\frac{a^{\prime}}{a}\left(\frac{a^{\prime}}{a}+\frac{n^{\prime}}{n}\right)-\frac{b^{2}}{n^{2}}\left(\frac{\dot{a}}{a}\left(\frac{\dot{a}}{a}-\frac{\dot{n}}{n}\right)+\frac{\ddot{a}}{a}\right)-k \frac{b^{2}}{a^{2}}\right\} .
\end{aligned}
$$

In the above expressions, a prime stands for a derivative with respect to $y$, and a dot for a derivative with respect to $\tau$.

The stress-energy-momentum tensor can be decomposed into two parts,

$$
\tilde{T}_{B}^{A}=\left.\check{T}_{B}^{A}\right|_{\text {bulk }}+\left.T_{B}^{A}\right|_{\text {brane }},
$$

where $\left.\check{T}_{B}^{A}\right|_{\text {bulk }}$ is the energy momentum tensor of the bulk matter, which will be assumed in the present work to be of the form

$$
\left.\check{T}_{B}^{A}\right|_{\text {bulk }}=\operatorname{diag}\left(-\rho_{B}, P_{B}, P_{B}, P_{B}, P_{T}\right),
$$

where the energy density $\rho_{B}$ and pressures $P_{B}$ and $P_{T}$ are independent of the coordinate y. Later, we will be specially interested in the case of a cosmological constant for which $-\rho_{B}=P_{B}=P_{T}$.

The second term $\left.T_{B}^{A}\right|_{\text {brane }}$ corresponds to the matter content in the brane $(y=0)$. Since we consider here only strictly homogeneous and isotropic geometries inside the brane, the latter can be expressed quite generally in the form

$$
\left.T_{B}^{A}\right|_{\text {brane }}=\frac{\delta(y)}{b} \operatorname{diag}\left(-\rho_{b}, p_{b}, p_{b}, p_{b}, 0\right),
$$

where the energy density $\rho_{b}$ and pressure $p_{b}$ are independent of the position inside the brane, i.e. are functions only of time. We may also add some other brane sources with similar energy momentum tensor. 
The assumption that $\tilde{T}_{05}=0$, which physically means that there is no flow of matter along the fifth dimension, implies that $\tilde{G}_{05}$ vanishes. It then turns out, remarkably, that the components $(0,0)$ and $(5,5)$ of Einstein's equations (3) (with (5) and (8)) in the bulk, can be rewritten in the simple form

$$
\begin{aligned}
F^{\prime} & =\frac{2 a^{\prime} a^{3}}{3} \kappa^{2} \check{T}_{0}^{0}, \\
\dot{F} & =\frac{2 \dot{a} a^{3}}{3} \kappa^{2} \check{T}^{5}{ }_{5},
\end{aligned}
$$

where $F$ is a function of $\tau$ and $y$ defined by

$$
F(\tau, y) \equiv \frac{\left(a^{\prime} a\right)^{2}}{b^{2}}-\frac{(\dot{a} a)^{2}}{n^{2}}-k a^{2} .
$$

Since $\check{T}_{0}^{0}=-\rho_{B}$ is here independent of $y$, one can integrate (12), which gives

$$
F+\frac{\kappa^{2}}{6} a^{4} \rho_{B}+\mathcal{C}=0,
$$

where $\mathcal{C}$ is a constant of integration which a priori depends on time. Assuming in addition that $\check{T}_{0}^{0}=\check{T}_{5}^{5}$, one finds using the time derivative of (12) and the $y$-derivative of (13) that $\rho_{B}$ is constant in time. This also implies that $\mathcal{C}$ is constant in time. In order to deal with the last component of Einstein's equations (see (6)), it is convenient to use the Bianchi identity,

$$
\nabla_{A} \tilde{G}^{A 0}=0,
$$

which can be rewritten (using $\tilde{G}_{05}=0$, and $\tilde{T}_{0}^{0}=\tilde{T}_{5}^{5}$ ) in the form

$$
\partial_{\tau}\left(\frac{F^{\prime}}{a^{\prime}}\right)=\frac{2}{3} \dot{a} a^{2} \gamma_{j}^{i} \tilde{G}_{i}^{j} .
$$

One finds (using (12)) that this equation is identically satisfied if $-\rho_{B}=P_{B}$. Hence, when the bulk source is a cosmological constant, any set of functions $a$, $n$, and $b$ satisfying (15) or, more explicitly,

$$
\left(\frac{\dot{a}}{n a}\right)^{2}=\frac{1}{6} \kappa^{2} \rho_{B}+\left(\frac{a^{\prime}}{b a}\right)^{2}-\frac{k}{a^{2}}+\frac{\mathcal{C}}{a^{4}},
$$

together with $\tilde{G}_{05}=0$, will be solution of all Einstein's equations (3), locally in the bulk.

The brane can then be taken into account by using the junction conditions [19], which simply relate the jumps of the derivative of the metric across the brane to the stress-energy tensor inside the brane. This procedure is described in detail 
in our previous paper [9] in the context of five-dimensional brane cosmology (see also [14]). The relevant expressions are

$$
\begin{aligned}
\frac{\left[a^{\prime}\right]}{a_{0} b_{0}} & =-\frac{\kappa^{2}}{3} \rho_{b}, \\
\frac{\left[n^{\prime}\right]}{n_{0} b_{0}} & =\frac{\kappa^{2}}{3}\left(3 p_{b}+2 \rho_{b}\right),
\end{aligned}
$$

where the subscript 0 for $a, b, n$ means that these functions are taken in $y=0$, and $[Q]=Q\left(0^{+}\right)-Q\left(0^{-}\right)$denotes the jump of the function $Q$ across $y=0$.

Assuming the symmetry $y \leftrightarrow-y$ for simplicity, the junction condition (19) can be used to compute $a^{\prime}$ on the two sides of the brane, and by continuity when $y \rightarrow 0$, (18) will yield the generalized (first) Friedmann equation (after setting $n_{0}=1$ by a suitable change of time):

$$
\frac{\dot{a}_{0}^{2}}{a_{0}^{2}}=\frac{\kappa^{2}}{6} \rho_{B}+\frac{\kappa^{4}}{36} \rho_{b}^{2}+\frac{\mathcal{C}}{a_{0}^{4}}-\frac{k}{a_{0}^{2}} .
$$

The salient features of this equation are that, first, the bulk energy density enters linearly, second, the brane energy density enters quadratically, and finally the cosmological evolution depends on a free parameter $\mathcal{C}$ (related to the choice of initial conditions in the whole space-time), whose influence corresponds to an effective radiation term (from the standard point of view, i.e. interpreting linearly the additional term) One can note that this equation is a generalization of the exact equation obtained in our previous paper [9]. This equation is enough to study the cosmological evolution in the brane, independently of the metric outside and in particular of the time evolution of $b$. The analysis of this equation will be postponed until section 4 . In the next section we will be interested in obtaining an explicit solution for the whole five dimensional metric.

\section{Explicit dependence on the fifth dimension for a stabilized bulk}

In this section, it will be shown that, with the help of an additional assumption, namely that the fifth dimension is static, in the sense that

$$
\dot{b}=0,
$$

\footnotetext{
${ }^{2}$ From a technical point of view, the presence of a free parameter, in contrast to the standard cosmological equations, can be explained in the following way. In four-dimensional cosmology, the component $(0,0)$ of Einstein's equations yields a first integral, because it contains only first derivatives in time. In the present work, we have used the component $(0,0)$ to integrate in $y$, whereas the time integration is operated with the help of the component $(5,5)$ which contains second derivatives in time.
} 
it is then possible to solve the full space-time metric, i.e. to determine the explicit dependence of the metric on the coordinate $y$. The restriction (22) allows us to go to the gauge

$$
b=1 .
$$

It then follows immediately from equation $\tilde{G}_{05}=0$, that $n$ can be expressed in terms of $a$ according to the relation

$$
\frac{\dot{a}}{n}=\alpha(t),
$$

where $\alpha$ is a function that depends only on time (and not on $y$ ). Inserting this into (12) yields the following differential equation:

$$
\alpha^{2}+k-\left(a a^{\prime}\right)^{\prime}=\frac{\kappa^{2}}{3} \rho_{B} a^{2},
$$

which is valid everywhere in the bulk (but not in the brane) on the two sides of the brane separately. It can be integrated in $y$, yielding

$$
a^{2}=A \cosh (\mu y)+B \sinh (\mu y)+C,
$$

with

$$
\mu=\sqrt{-\frac{2 \kappa^{2}}{3} \rho_{B}},
$$

in the case $\rho_{B}<0$, or

$$
a^{2}=A \cos (\mu y)+B \sin (\mu y)+C,
$$

with

$$
\mu=\sqrt{\frac{2 \kappa^{2}}{3} \rho_{B},}
$$

in the case $\rho_{B}>0$, or finally

$$
a^{2}=\left(\alpha^{2}+k\right) y^{2}+D y+E,
$$

for $\rho_{B}=0$. In the following, we will focus on the first case $\rho_{B}<0$, but all the equations will apply as well to the case $\rho_{B}>0$, up to the transformation $\mu \rightarrow i \mu$, $B \rightarrow-i B$.

The coefficients $A, B, C, D, E$ are functions of time, and $C$ is expressible in terms of $\alpha$ as

$$
C=3 \frac{\alpha^{2}+k}{\kappa^{2} \rho_{B}},
$$

and the others can be determined by the junction conditions. The symmetry $y \leftrightarrow-y$ imposes the relations $A_{+}=A_{-} \equiv \bar{A}, B_{+}=-B_{-} \equiv \bar{B}$ between the coefficients on the two sides of the brane. Using (19) and (20), one then finds

$$
\frac{\mu \bar{B}}{\bar{A}+C}=-\frac{\kappa^{2}}{3} \rho_{b}, \quad \frac{2 \mu \dot{\bar{B}}}{\dot{\bar{A}}+\dot{C}}=\kappa^{2}\left(p_{b}+\frac{1}{3} \rho_{b}\right) .
$$


Note that one can check explicitly energy conservation in the brane from these relations, i.e.

$$
\dot{\rho}_{b}+3 \frac{\dot{a}_{0}}{a_{0}}\left(\rho_{b}+p_{b}\right)=0 .
$$

Let us finally fix our temporal gauge, by imposing the condition $n_{0}=1$, i.e. we choose as time what corresponds to the cosmic time in the brane. The function $\alpha$ is then simply $\dot{a}_{0}$ (see equation (24)), and (26) specialized to $y=0$ gives

$$
\bar{A}=a_{0}^{2}-C,
$$

with, from equations (31) and (21),

$$
C=\frac{a_{0}^{2}}{2}\left(1+\frac{\kappa^{2} \rho_{b}^{2}}{6 \rho_{B}}\right)+\frac{3 \mathcal{C}}{\kappa^{2} \rho_{B} a_{0}^{2}} .
$$

Using the first relation in (32), the remaining coefficient is determined as

$$
\bar{B}=-\frac{\kappa^{2} \rho_{b}}{3 \mu} a_{0}^{2}
$$

Substituting back the coefficients $A$ and $B$ thus obtained in the general expression (26) and using (21), one finally obtains the following expression for the scale factor $a(t, y)$ :

$$
\begin{aligned}
a(t, y)= & \left\{\frac{1}{2}\left(1+\frac{\kappa^{2} \rho_{b}^{2}}{6 \rho_{B}}\right) a_{0}^{2}+\frac{3 \mathcal{C}}{\kappa^{2} \rho_{B} a_{0}^{2}}\right. \\
& +\left[\frac{1}{2}\left(1-\frac{\kappa^{2} \rho_{b}^{2}}{6 \rho_{B}}\right) a_{0}^{2}-\frac{3 \mathcal{C}}{\kappa^{2} \rho_{B} a_{0}^{2}}\right] \cosh (\mu y) \\
& \left.-\frac{\kappa \rho_{b}}{\sqrt{-6 \rho_{B}}} a_{0}^{2} \sinh (\mu|y|)\right\}^{1 / 2} .
\end{aligned}
$$

The other coefficient of the metric, $n(t, y)$, follows directly from the above expression with the help of the relation (24), i.e.

$$
n(t, y)=\frac{\dot{a}(t, y)}{\dot{a}_{0}(t)} .
$$

Therefore, from the two functions $a_{0}(t)$ and $\rho_{b}(t)$ restricted to the brane, which are obtainable by solving the coupled system constituted of the two equations (21) and (33), one is able to infer the extension of the metric in the bulk away from the brane.

Let us mention briefly the case $\rho_{B}=0$. Following the same procedure as above, one would end up with the following expression for the scale factor:

$$
a(t, y)=\left\{a_{0}^{2}-\frac{\kappa^{2} \rho_{b}}{3} a_{0}^{2}|y|+\left\{\frac{\kappa^{4} \rho_{b}^{2}}{36} a_{0}^{2}+\frac{\mathcal{C}}{a_{0}^{2}}\right\} y^{2}\right\}^{1 / 2} .
$$


In the special case $\mathcal{C}=0$, one recognizes the linear solutions obtained in our previous paper [9].

We have not specified here whether the fifth dimension is compact or not. If it is compact, then one must check that one gets in the end a globally well defined solution (see [9]).

The next section will be devoted to the determination of the evolution of the scale factor on the brane, $a_{0}(t)$, using equations (21) and (33), which - we wish to emphasize - does not depend on the hypothesis (22) of the present section.

\section{Cosmological scenarios}

In this section we will examine the consequences of equation (21) on the cosmological evolution inside the brane. In general, one still finds the quadratic behaviour in the brane energy density which leads to a non conventional cosmology [9]. However, as suggested by [11] and [12], we will show that one can indeed implement the idea of Randall and Sundrum [6] in the cosmological context to recover standard cosmology. We will find exact solutions that match the early non conventional cosmology to the subsequent ordinary cosmology.

Let us thus assume that the energy density in the brane can be decomposed into two parts,

$$
\rho_{b}=\rho_{\Lambda}+\rho,
$$

where $\rho_{\Lambda}$ is a constant that represents an intrinsic tension of the brane and $\rho$ stands for the ordinary energy density in cosmology. Substituting in (21) one gets

$$
\frac{\dot{a}_{0}^{2}}{a_{0}^{2}}=\frac{\kappa^{2}}{6} \rho_{B}+\frac{\kappa^{4}}{36} \rho_{\Lambda}^{2}+\frac{\kappa^{4}}{18} \rho_{\Lambda} \rho+\frac{\kappa^{4}}{36} \rho^{2}+\frac{\mathcal{C}}{a_{0}^{4}}-\frac{k}{a_{0}^{2}} .
$$

If we follow Randall and Sundrum by choosing $\rho_{\Lambda}$ such that

$$
\frac{\kappa^{2}}{6} \rho_{B}+\frac{\kappa^{4}}{36} \rho_{\Lambda}^{2}=0
$$

then one sees that standard cosmology is recovered with the identification ([11], [12])

$$
8 \pi G \simeq \frac{\kappa^{4} \rho_{\Lambda}}{6}
$$

when $\rho \ll \rho_{\Lambda}$. Let us however keep $\rho_{\Lambda}$ unspecified at this stage. In the case $\rho=0$ and $\mathcal{C}=0$ one would recover the solutions given by Kaloper 13.

To get analytic solutions we will now assume that ordinary matter is described by an equation of state of the form $p=w \rho$, with $w$ constant. Then, using equation (33) for $\rho$ and $p$, one can write

$$
\rho=\rho_{*}\left(a_{0} / a_{*}\right)^{-q}, \quad q=3(1+w),
$$


where $\rho_{*}$ and $a_{*}$ are constants. Inserting this expression in (41), it is possible to integrate explicitly the resulting equation in the case where $\mathcal{C}=0$ and $k=0$. Assuming the first term on the right hand side of (41) to be positive and defining

$$
\lambda=\sqrt{\frac{\rho_{B}}{6 \kappa^{2}}+\frac{\rho_{\Lambda}^{2}}{36}},
$$

one finds, for $\lambda>0$,

$$
a_{0}=a_{*} \rho_{*}^{1 / q}\left\{\frac{\rho_{\Lambda}}{36 \lambda^{2}}\left[\cosh \left(q \kappa^{2} \lambda t\right)-1\right]+\frac{1}{6 \lambda} \sinh \left(q \kappa^{2} \lambda t\right)\right\}^{1 / q},
$$

and, for $\lambda=0$,

$$
a_{0}(t)=a_{*}\left(\kappa^{2} \rho_{*}\right)^{1 / q}\left(\frac{q^{2}}{72} \kappa^{2} \rho_{\Lambda} t^{2}+\frac{q}{6} t\right)^{1 / q}
$$

(the origin of time being chosen so that $a_{0}(0)=0$ ). It is clear, from the latter expression, in the case $\lambda=0$, how one passes from a very early universe, characterized by a non-conventional evolution $a(t) \sim t^{1 / q}$, to a late time phase described by standard cosmology, $a(t) \sim t^{2 / q}$. In the case $\lambda>0$, with $\lambda$ sufficiently small, one obtains three successive phases, a non-conventional phase dominated by $\rho^{2}$, a conventional phase dominated by $\rho$ and, finally, an exponential phase, where $\lambda$ plays the rôle of an effective cosmological constant in our Universe. Therefore, brane cosmology is able to produce a late time acceleration phase such as seems to be needed from the latest cosmological observations (20]), at the price however of a tiny mismatch in the compensation between $\rho_{B}$ and the tension of the brane. The required fine-tuning expresses the brane cosmological version of the well-known cosmological constant problem.

Let us finally examine the case of a universe filled with radiation $(w=1 / 3)$. It is then possible, still with $k=0$, to integrate explicitly the case $\mathcal{C} \neq 0$, because the $\mathcal{C}$-term has the same dependence on $a_{0}$ as the term proportional to $\rho$ in (41). Moreover, the free parameter $\mathcal{C}$ can be constrained by nucleosynthesis. Indeed, at the time of nucleosynthesis, the universe is dominated by the radiation energy density, which can be written

$$
\rho_{\text {rad }}\left(t_{N}\right)=g_{*} \frac{\pi^{2}}{30} T_{N}^{4}
$$

where $g_{*}$ is the effective number of relativistic degrees of freedom at that time. In the standard model, $g_{*}($ standard $)=10.75$, and any deviation $\Delta g_{*}$ is strongly constrained by the observed abundances of light elements, typically $\Delta g_{*}<2$. In our model, since the additional $\mathcal{C}$-term evolves like radiation, it can also be seen effectively as additional relativistic degrees of freedom, subject to the usual constraint, so that

$$
\rho_{\mathcal{C}}\left(t_{N}\right) \equiv \frac{3 \mathcal{C}}{8 \pi G a^{4}\left(t_{N}\right)} \leq \frac{\pi^{2}}{15} T_{N}^{4}
$$


at nucleosynthesis.

To conclude, we wish to recall the main results of this work. First, it has been shown that one can obtain a first integral of Einstein's equations, which provides, on the brane, a relation analogous to the (first) Friedmann equation and which depends only on the geometry and matter content of the brane, except

for a constant parameter. Second, when $\dot{b}=0$, one can extend explicitly the solution found on the brane to the whole spacetime. Finally, we have shown, exhibiting exact solutions, that brane cosmology appears compatible, at late times, with standard cosmology, in a Randall-Sundrum type model. In this respect, an important question for future investigations is wether an (exact or quasi) RandallSundrum configuration can be naturally reached in a dynamical scenario for the very early universe.

Note added: Several papers which discuss related issues have appeared quasi simultaneously to this one [21, 22] (see also [23]).

\section{References}

[1] D. Bailin, A. Love, Rep. Prog. Phys. 50 (1987) 1087.

[2] N. Arkani-Hamed, S. Dimopoulos, G. Dvali, Phys.Lett. B429, (1998) 263, and hep-ph/9807344; I. Antoniadis, N. Arkani-Hamed, S. Dimopoulos, G. Dvali, Phys. Lett. B436, 257 (1998).

[3] N. Arkani-Hamed, S. Dimopoulos and G. Dvali, Phys. Rev. D59 (1999) 086004 .

[4] M. Gogberashvili, hep-ph/9812296; hep-ph/9812365; hep-ph/9904383.

[5] L. Randall, R. Sundrum, Phys. Rev. Lett. 83, 3370 (1999) hep-ph/9905221].

[6] L. Randall, R. Sundrum, Phys. Rev. Lett. 83, 4690 (1999) hep-th/9906064.

[7] V.A. Rubakov, M.E. Shaposhnikov, Phys.Lett.B125,139 (1983); M. Visser, Phys.Lett.B159,22 (1985); E.J. Squires, Phys.Lett. B167, 286 (1986); G.W. Gibbons, D.L. Wiltshire, Nucl. Phys. B287, 717 (1987).

[8] H. Davoudiasl, J.L. Hewett, T.G. Rizzo, hep-ph/9909255; D.E. Krause, E. Fischbach, hep-ph/9912276.

[9] P. Binétruy, C. Deffayet, D. Langlois, hep-th/9905012, Nucl. Phys. B., in press. 
[10] P.Kanti, I.I. Kogan, K.A. Olive, M. Pospelov. UMN-TH-1820-99, hep$\mathrm{ph} / 9909481$

[11] C. Csáki, M. Graesser, C. Kolda, J. Terning, hep-h/9906513.

[12] J.M. Cline, C. Grosjean, G. Servant, hep-ph/9906523.

[13] N. Kaloper, hep-th/9905210.

[14] H.A. Chamblin, H.S. Reall, hep-th/9903225.

[15] T. Nihei, hep-ph/9905487; H.B. Kim, H.D. Kim, hep-th/9909053.

[16] M. Cvetic, H.H. Soleng, Phys. Rept. 282, 159 (1997).

[17] A. Lukas, B.A. Ovrut, K.S. Stelle, D. Waldram, Phys.Rev.D59, 086001 (1999); A. Lukas, B.A. Ovrut, D. Waldram, hep-th/9902071.

[18] U. Ellwanger, hep-th/9909103.

[19] W. Israel, Nuovo Cimento B 44 (1966).

[20] A.G. Riess et al., Astroph. J 116, 1009 (1998); S. Perlmutter et al., "Measurements of Omega and Lambda from 42 High-Redshift Supernovae", astroph/9812133.

[21] T. Shiromizu, K. Maeda, M. Sasaki, gr-qc/9910076.

[22] E.E. Flanagan, S.-H.H. Tye, I.Wasserman, hep-ph/9910498.

[23] P. Kraus, JHEP 9912 (1999) 011; A. Kehagias, E. Kiritsis, hep-th/9910174. 